\title{
A Study on Screen Translation Criteria from the Perspective of the Audience
}

\author{
Ying Sun \\ School of Foreign Studies, Northwestern Polytechnical University, China
}

Copyright $\bigcirc 2019$ by authors, all rights reserved. Authors agree that this article remains permanently open access under the terms of the Creative Commons Attribution License 4.0 International License

\begin{abstract}
Under the background of globalization, the society has seen an increasing need for cultural exchange. As bridges between different languages, translations are needed for different occasions. Screen translation is a relatively new discipline in translation studies. Especially with the booming of the TV shows in Western countries, which have gained popularity all over the world, screen translation studies become more necessary. This paper discusses the features of subtitling and dubbing translation from the audience's perspective, and the ultimate purpose of this study is to formulate a set of criteria for screen translation in general, based on these features.
\end{abstract}

Keywords Screen Translation, Subtitling, Dubbing, Criteria

\section{Introduction}

In the past few decades, the society has seen an increasing need for political, economical and cultural communication between countries. To conquer the language barriers, translation studies all around the world boomed. Under this rapid growth, not only the range of translation is expanded, but also its forms multiplied. It becomes more evident that translation is no longer an isolated discipline but part of a bigger one. Interdisciplinary studies such as medical translation, legal translation, and screen translation comes into being.

Screen translation (ST), in particular, is an even newer discipline which involves much more than merely the verbal translation. Nowadays, under the economic globalization, the world has witnessed an increasing amount of film and television program exchanges between countries, making the studies on the translation of these audiovisual materials more necessary than ever. Yet studies on ST is not abundant, and even fewer for studies on criteria for ST. A set of comprehensive criteria can provide better guidance for ST translators.

\section{Theoretical Framework}

Writing from the audience's point of view, the author uses three audience-related theories in the analyzing process, namely, reception theory, psycholinguistics and cognitive psychology.

Since this study centers on the receiving end of ST, i.e., the audience and their response, reception theory is chosen as the main theory for this paper. In fact, it lays the theoretical foundation for the analysis of the whole paper. Psycholinguistics is also adopted, for it studies how language is understood by people. And cognitive psychology assists the study by providing valuable theoretical evidences in the analysis of the receiving process of ST.

\subsection{Reception Theory}

Reception theory, also called Rezeptionsästhetik (reception aesthetics), or reader-response theory, originated in Germany at the end of the 1960s. Reception theory is not a unified body of theory but represents a rather large field of discussion. Since it was represented by five professors at Konstanz University in the south of Germany, Konstanz School was later used to refer to reception theory. It is more or less the German equivalent of the American term "Reader-Response Criticism" and is particularly associated with the names of Hans Robert Jauß whose book Towards an Aesthetic of Reception appeared in 1982[1], and Wolfgang Iser, who published his The Act of Reading: a Theory of Aesthetic Response in 1987[2]. Reception theory emphasizes on the reader rather than the text or the author. It seeks empirical evidence, through historical or ethnographic research, that documents the production and circulation of meaning. "The readers' receptions of a text are completely independent from the author's original intentions or the literary critics' interpretations of the text. The meaning of a text is defined by its readers' receptions: readers, not the authors, make meaning. "[3]" There is thus not a single pre-determined 'adequate' reception of a given text on which literary theory needs to focus. Instead, all 
actual receptions in the past and the present are valid as such, and their particular characteristics become the objects of study for a 'reception history'. "[4]

According to reception theory, a literary work is full of "indeterminacies " or "gaps". They are the basic elements in literature works that cause the reader's acceptance and response, and are "a bridge between the writer's and reader's consciousness. " [5] Thus a kind of interaction between the writer and the reader (either between the original writer and the translator or between the translator and the actually reader) is achieved. There is also a relationship between text and reader (either between the original text and the translator or between the translated text and the actually reader). If the text is closer to reader's aesthetic experience, it can be easily accepted. On the contrary, if too far away from reader's aesthetic experience, it will be hard to accept. Reception theory called this "aesthetic distance." Jauß also stated that each individual has his or her own unique "horizon of expectation," which designates an area of "collective" assumptions, genre conventions and cultural ideologies shared by texts and readers. [1] If the text being read shares the same "horizon" with the readers, the "aesthetic distance" is closer, but it may lose the interest of them, but if it exceeds the "horizon" of the readers, it may leave them greater space for imagination but might become harder to understand. Therefore, "horizon of expectation" becomes a good yardstick of "aesthetic distance." But reception theory also argues that whether to shorten or maintain the distance between the "horizon" of original text and target readers depends on whether the readers are able to understand the translated text. That is to say, if translators want to make the translated text easier to be accepted or understood by readers, their "horizon of expectation " should fuse with the readers' as much as possible. This explains many of the decisions made in the translation of cultural elements in audiovisual materials.

Screen translation, among all different types of translation, benefits the most from reception theory. As we know, the audience is the ultimate receivers of the audiovisual products. These products are made for the audience and their acceptance in turn decides the fate of the products. That is to say, putting the readers-the audience-in the central place is especially important in ST. If the translation of the imported films or TV programs is not satisfactory to the audience, it may lose the interest of them even though the films or TV programs themselves are good. And the importers may wind up having financial losses. Reception theory, which directly deals with the response of the audience, is therefore appropriate to be used in the analysis of ST.

\subsection{Psycholinguistic Studies}

As an emerging interdisciplinary science, psycholinguistics focuses on the overlapping area in psychology and linguistics. Based on theories of psychology and linguistics, psycholinguistics adopts the research methodology of psychology to work out the relationship between language and psychological processes so as to find out the rules governing different language activities.

There are three major issues psycholinguistics tries to solve: the psychological process of speech generation, or the process of translating ideas to be expressed into actual speech utterance; the psychological process of speech comprehension, or the process of decoding the ideas conveyed in the speech signs auditorily or visually perceived; and the process and mechanism of language acquisition. [6] Among the three issues, the second one is clearly one of the main concerns in ST since it deals directly with the audience perception of the translation of the audiovisual materials, be it in subtitling or dubbing form.

In ST, how subtitles or dubbings are understood is crucial to the understanding of the film. This is also why psycholinguistics can be applied in the study of ST. Since film language is transient, the decoding process must take as less time as possible. Subtitles being too long, the audience may only have time to decode the first part and miss the rest. Since everyone's vocabulary repertoire varies, the retrieving of the word one reads or hears may take different time for different people. Even the same person may have different listening and reading vocabulary repertoires. What can be easily decoded by listening may turn out to be difficult by reading. For some people, certain complicated words may not even be decodable. It is the same with dubbing translation. Unfamiliar or archaic words may take too much time to decode, so more colloquial words are usually chosen in dubbing translation (except when the film requires the translation to be more formal). Psycholinguistics can also be used to explain the translation of cultural images. Some original cultural information may not be retrievable by the target audience, so adaptations need to be made.

\subsection{Cognitive Psychology}

Cognitive psychology is one of the more recent additions to psychological research, having only developed as a separate area within the discipline since the late 1950s and early 1960s (though there are examples of cognitive thinking from earlier researchers). According to cognitive psychology, human brain shares many similarities with computer in functional structure and information processing. So what cognitive psychology does is to use computer as the model of human psychology, and to make a unified explanation of both human psychology and computer behavior so that the principia of information processing in human brain can be found. [7] According to Wang Su, a professor of cognitive psychology, so far, on the general principia of information processing, 
psychologists Newell and Simon have made the most comprehensive explanation. They believe both human and computer's information processing system includes receptor, effector, memory and processor. Receptor receives information and effector responds to it. Information processing system uses symbol structures to indicate input and output. Memory can store or retrieve these symbol structures. [8]

Cognitive psychology is chosen for this paper because it can be used to study the mental process of the audience. This fits the general audience-oriented direction of the paper. Cognitive psychology is mainly used to analyze the receiving process of the audience, which consists of several stages.

\section{General Introduction to Screen Translation}

\subsection{The Characteristics of Film Language}

The uniqueness of ST lies largely in the unique nature of film language. Even though the translation of film language falls under the general guidance of translation theories and methodologies, the special characteristics of film language determine that special rules need be applied to its translation.

To begin with, films are multi-dimensional; therefore, any film is polysemiotic in texture [9]. According to Pavlović, what TV audience receives via the audio-visual medium is a text consisting of a variety of audio and visual, and verbal and non-verbal components, all of which stand in a complex relationship with one and another [10]. Gottlieb reiterated that "a screen adaptation of a 100,000 word novel may keep only 20,000 words for the dialogue, leaving the semantic load of the remaining 80,000 words to the non-verbal semiotic channels - or to deletion" [11]. This polysemiotic nature of film language makes film translation a testing task. Some even consider it impossible. With this unique feature of film language in mind, researchers need to be aware that the study of screen translation can never be constrained merely to the verbal level.

Compared with written literature language, film language is audio and visual in nature, and its receiver is the general public, which determines that, film language is, in most cases, informal rather than formal, and colloquial rather than literary. Targeting at people of all social status, it is usually closer to life and culturally loaded [12].

However, not all film language is colloquial. Take the film The Lord of the Rings for example. The lines throughout the film are all quite formal, even poetic. This brings out another feature of film language - variability. Film language can vary with different film genres, and even with different film characters - characters from different social status and walks of life.
Film is, in essence, a reflection of real life. However, to make the conveyed meaning to be appreciated more easily by the audience, film is usually a more dramatic reflection of life. Every tiny detail is magnified, and every aspect of life is portrayed dramatically, even in exaggeration, so that the audience feels what the actors feel, laughs what they laugh, and cries what they cry. Therefore, the language of film is more often than not, quite dramatic.

Last but not the least, unlike written language in literature works, film language is all transient. "[...] the audience is given but one chance to understand the information that comes across to them" [9]. So when it disappears, it cannot be retrieved. Even though DVDs and VCRs make re-reading possible, it will be too troublesome and time-consuming to keep rewinding and backtracking what has been said.

Qian shaochang once concluded that film language is audio, comprehensive, transient, colloquial, and noteless in nature [13]. These characteristics corresponds to most of the features the author has described above. Though detailed, the author believes that some improvements can be made. Based on Prof. Qian shaochang's five language characteristics, the author is hereby making some further adaptations, and concluded them into seven characteristics:

- polysemiotic (instead of "comprehensive"): the language consists of a variety of audio and visual, and verbal and non-verbal components;

- audio and visual (instead of "audio"): the language is audio or visual in form instead of written;

- colloquial: the language is more spoken than written, and more informal than formal. It is closer to life;

- variable: the language can vary with different film genres, and with different film characters;

- dramatic: the language is a dramatic reflection of life, some even in exaggeration;

- transient: the language is gone once it is spoken;

- noteless: the language is usually spoken and a note cannot be added.

\subsection{Characteristics and Constraints of the Two Main Types of Screen Translation}

\subsubsection{Subtitling Translation}

Subtitling translation is a complex process which involves switching speech in one language into written form in another. Gottlieb described this mode switch from speech to writing as diasemiotic [11]. Baker also explained at length that when translating film and television programs, four simultaneous channels need to be taken into consideration. They are: the verbal auditory channel (dialogue, background voices, and sometimes lyrics), the non-verbal auditory channel (music, natural sound and sound effects), the verbal visual channel (superimposed titles and written signs on the screen) and the non-verbal visual channel (picture composition and flow). In subtitling translation, the balance is shifted from the verbal auditory 
channel to verbal visual channel [14]. Because the translation is in written form, certain features of speech such as intonations cannot be demonstrated in the subtitles. The image of the film (non-verbal visual channel) should also be considered in translation because subtitles are now part of it.

However, this is not the only constraint subtitling translator faces. Gottlieb spoke of formal (or quantitative) and textual (or qualitative) constraints on subtitling [15]. The former are imposed on the subtitles by the visual context of the film, and the latter are space and time factors. The latter, also called spatio-temporal constraints, are believed to be the most important factors that constrain the translators.

There are several reasons that contribute to its importance. First of all, subtitling translation is limited by the size of the TV screen. Subtitles are usually provided at the bottom of the screen with a maximum of two lines so that the image of the film will not be blocked. In addition, even within this limited space of screen, certain space on the left and right (subtitles are usually centered) is required to facilitate the reading. However, it does not mean the larger the TV screen is, the longer the subtitles can be; but rather, if the TV screen is bigger, the font size of the subtitles increases, and the space problem remains. The font size of subtitles needs to be carefully chosen. Too large, it may take up too much space and worsen the space crisis; and too small, it may impede the reading of the audience.

Secondly, subtitling translation is also restrained by time or the duration of utterance. Depending on the speed at which the material is spoken, the time for display usually lasts from half a second to three seconds, and intervals are needed, or else the audience will not be able to differentiate the ending of one subtitle from the beginning of another one. These two elements, together with the average viewer's reading speed and the demand for an informative subtitling, construct a huge barrier for the translation of subtitles.

And thirdly, under the limitation of time and space, cultural elements deteriorate the situation, for there is simply no time to explain the cultural knowledge in the subtitles.

To summarize in Fawcett's words, the subtitles' work sheets are "messy, with doubts and hesitations, flashes of insight and blind spots, as the translator searches for the equivalences which will fit the constraints of each given situation" [16].

\subsubsection{Dubbing Translation}

Dubbing translation, even though involves no mode switch, is actually even more complicated than subtitling, for it usually involves the meticulous work of a group of people. And there can be a number of additional factors that contribute to the quality of dubbing, such as "the use of up-to-date equipment, the choice of actors and the skill of the editor and the sound engineering" [17]. Other factors may include: compatibility of source and target language, the budget available to fund what is quite a complex and time-consuming operation, the skill of the translator(s), especially in capturing the appropriate tone and register and, last but not the least, the ability of the producer to coax the best possible performance out of his/her team of actors. [18]

As far as the four channels are concerned, in dubbing, where foreign-language dialogue is replaced by domestic-language dialogue, the audiovisual balance is maintained: the four semiotic channels each keep their original semantic load [14].

Since dubbing is a replacement of the original soundtrack with the new one while the film image remains the same, lip synchronization is inevitable. Even though an exact match is impossible, the translators need to try their best to convince the audience that the actors are speaking another language. That means dubbing starts when the actor utters the first word and ceases when s/he finishes. This is the temporal constraint in dubbing translation.

The second is the lip movement constraint. The new voice should match the actors' lip movements as closely as possible, especially in close-ups. That is to say, the genre the film or TV program belongs to also poses challenge on dubbing translation for certain genres such as court films tend to use more close-ups than the others.

The third constraint derives from the need to match the utterance of the dubbed actors and their actions on the screen. A mismatch of the two can easily annoy or even confuse the audience. This constraint means careful adjustments must be made in the word or sentence order so that the lines fit the film image better.

These temporal, lip-sync and sound-image sync imperativeness seriously constrain the dubbing translation. And again the complex situation is made worse by cultural elements, for there is simply no time to explain the cultural images in dialogues.

To summarize, dubbing translation is a complicated process which requires studious adjustment of words and sentences to make the dubbed version sound more natural. As Kilborn described it, "[...] with dubbing the art is to hide the art" [18]. And in Bakewell, a dubbing practitioner's words: The ideal end-product would be the perfect illusion. The best possible response from the audience would be for them never to be aware that we had done anything at all. Dubbing, after all, is the art of being totally inconspicuous [19].

As can be seen from above, subtitling and dubbing translation are vastly different in many aspects. That means the usual practice of treating them in the same way is, to a large degree, inappropriate. Given the differences, the author would like to make some suggestions on the different strategies that could be employed in subtitling and dubbing translation respectively: 
1. Simplification should be used in subtitling translation while the synchronization of the time of utterance and lip movements should be employed in dubbing translation;

2. Verbal-image synchronization is suggested in subtitling. It is also required in dubbing;

3. Words of mood are mostly omitted in subtitling translation, but in dubbing, they need to be translated or substituted with word of the same length;

4. Language used in subtitling translation, more often than not, is more formal than that of dubbing but less formal than written, and dubbing translation needs to be more colloquial and domesticated;

5. Cultural elements can be briefly noted in subtitles, but domesticated in dubbing.

The five strategies are proposed to help screen translators to better deal with the constraints in both forms of translation, and to make the translated versions more acceptable to the audience.

However, besides their differences, subtitling and dubbing translation also share some features that are fundamentally the same. This makes the establishment of a set of screen translation criteria possible.

\section{Screen Translation Criteria}

Translation quality assessment (TQA) has always been one of the most discussed topics in translation field. According to Baker, "Approaches to TQA fall into a number of distinct categories: anecdotal and subjective, including neo-hermeneutic approaches; response-oriented approaches; and text-based approaches " [14].

The assessment of screen translation quality is equally important, for a bad subtitling work can always ruin a film. Nornes once humorously put it, "all of us have, at one time or another, left a movie theater wanting to kill the translator "[20]. This is because no matter how different subtitling and dubbing translation are, the translations inevitably produce a film version that to some degree compromises the original, no matter how conscientiously the translators have worked. So what is a good translation is ST? What are the criteria to say that one translated version is a "good" compared with another "bad" or "poor" one? German functionalists believe that translation criteria should be proposed according to different literary types. They believe there are two kinds of translation principles, general principles that fit all translation, and special principles used in special circumstances [21]. And ST definitely falls into the second category. So far, however, with so many criteria at hand, there is not yet a relatively complete set of criteria that fits specifically for screen translation. Yet one is needed because the existing criteria are either too general, not taking the unique nature of ST into account and only partially fit for ST, or incomplete, fitting the nature of ST but need to be supplemented with other criteria. A good example is Nida's four basic requirements of a translation, viz. making sense, conveying the spirit and manner of the original, having a natural and easy form of expression and producing a similar response. They fit ST well but need to be complemented.

On the ground of the criteria formulated by forerunners and the features and constraints of the two main types of screen translation, the author is hereby proposing a set of criteria that fits specially for ST, in the hope that it can be a guideline for screen translators and can shine some light upon the future ST studies. Since audience-related theories are the foundation of this paper, response-oriented approaches are used in the formulating of these criteria.

Altogether, the author proposes eight criteria for ST. In order to make it easier to remember, the author coins them into one word, FANCIFUL, with each letter representing one criterion, and the word "fanciful" itself is also a vivid display of the feature of film industry.

\subsection{The FANCIFUL Criteria of ST}

\subsubsection{F for Faithfulness}

Faithfulness is a criterion that almost appears in every set of standards in TQA, starting from Yan Fu's three criteria of faithfulness, expressiveness and elegance [22] to Gu Zhengkun's "(closely) pursuing faithfulness [23]." It can also be seen in Lin Yutang's "faithfulness, smoothness and beautifulness." [24] It is safe to say that faithfulness has become one of the most important professional ethics to translators.

In screen translation, faithfulness is also required so that the original intentions of the film can be expressed. Yet, because of the cultural elements in film language, re-creation is frequently used in ST to make the untranslatable translatable. This re-creation, however, does not contradict to the faithfulness criterion. Re-creation is on semantic level, but pragmatically speaking, its ultimate goal is still to make the translation faithful to the film scenario. In Nida's criteria, that is to produce a similar response in translation. So as long as the re-creation is properly based upon the scenario in which it is spoken, and the desired response expected from the original film creator is successfully obtained from the audience, it is considered faithful.

\subsubsection{A for Acceptability}

Acceptability here refers not to how well the translated version is understood but whether it can be accepted by the receivers. It is especially important in ST, for films, more than other forms of arts, carry a country's cultural beliefs, values and moral standards. What is acceptable in one culture might turn out to be a taboo in another. And the acceptance of a foreign film largely relies on the translation provided.

As argued by reception theory, any translation should first take the audience's emotional feelings and aesthetic 
judgments into consideration. The last thing a translator wants to do is to offend the audience in the target country because for any imported film, to have a good box office in the target country is the ultimate goal, and whether it is accepted by the audience directly affects the proceeds of it. A good example is the translation of foul words and sex-related words. The general practice of toning down the sex-related words and the vulgarities in foul words is for the acceptance of the audience.

\subsection{3. $\mathrm{N}$ for Naturalness}

Naturalness is also a frequently mentioned criterion in TQA. For example, Lin Yutang proposed "smoothness" [24] in his three criteria of "faithfulness, smoothness and beautifulness," and Nida also pointed out that translation need to have "a natural and easy form of expression." [25]

Naturalness in ST means that translation must sound like natural utterance of the target language, especially in dubbing translation. Awkwardness in ST can hinder the understanding of the audience, and thus slowing down the speed of their reading or listening comprehension process. Since the audience has but one chance to mentally process the information, naturalness is definitely needed. Some scholars use the term "invisibility" to refer to this naturalness. Jaskanen commented on the invisibility of subtitling, explaining that subtitling should not be obstructive to the eye and should ideally blend in with the film [26].

Domestication is also a way to create naturalness, shortening the distance between target audience and the film scenarios in an unfamiliar culture. It also further makes the film more acceptable to the target audience.

The task as subtitlers is to create subliminal subtitles so in sync with the mood and rhythm of the movie that the audience isn't even aware it is reading. We want not to be noticed. [27] This is also another way to create naturalness - synchronization, which is also our next criterion.

\subsection{4. $\mathrm{C}$ for $\mathrm{C}(\mathrm{S}) \mathrm{ynch}$ ronization}

Since "C" and "S" here share the same pronunciation, the author borrows the sound of " $\mathrm{C}$ " and uses it to refer to Synchronization.

Subtitles are a part of a film. They cannot exist alone. That means they must appear in sync with sound. [28] Synchronization is rarely mentioned as a criterion in the translation field. It is understandable because film language is different from any other type of language text in translation field, which makes ST a unique type of translation.

Synchronization, as we mentioned, is determined by the polysemiotic nature of film language. Here it refers not just to the lip-synchronization but to the verbal-image synchronization. Mismatch of verbal info and film image can cause great annoyance, even making it difficult to understand. Therefore, it should be used as a yardstick in the assessment of ST, for it fits the cognitive and psychological needs of the audience in the receiving process, which is to reduce their cognitive efforts when they correlate what they read/hear (the verbal info) with what they see on the screen (film image).

\subsubsection{I for Informality Degree}

Informality degree refers to the style of language that should be used in ST. The style used in translation should fit the style used by the original actors. This is what Nida referred to as "conveying the spirit and manner of the original." [25]

Since generally speaking, language in ST is more informal than other types of translation, the author here uses "informality degree" instead of "formality degree." Different people may speak differently due to their different social identities. In this sense, screen translators should always take this factor into account, and ask "how informal the language should be?" before making the final translation.

\subsection{6. $\mathrm{F}$ for Fun}

Fun here not necessarily means to make the audience laugh. It actually refers to the general entertainment of the audience. It is listed as a criterion because any film is aimed at pleasing the audience, regardless of whether the audience's reaction is amusement (comedy), fear (horror), sadness (tragedy), excitement (action) or anything else.

Re-creation is one of the best ways to achieve this, especially for the creating of humors. Conformed to the precondition of faithfulness, proper re-creation not only makes the untranslatable cultural difficulties translatable, but it also maintains the desired effects created by the original creator.

No matter which technique is used, translators should always try their best to entertain the audience the way the original film is supposed to do.

\subsection{7. $\mathrm{U}$ for Understandability}

Understandability refers to how well the target receiver can understand the translated version. Even though it is the seventh criterion proposed by the author, it is one of the most important criteria used in the judging of a translated version in ST. In fact, many theorists have mentioned it in their criteria, only in different wordings. Yan $\mathrm{Fu}$, for example, called it "expressiveness," [22] and Nida used "making sense. [25]"

Any translation work, in essence, is to help the readers understand what they originally cannot, so it is with ST. According to research in audience reception psychology, "the postmodern viewers' desire for 'depthless' calls for a direct and superficial visual culture" [29]. So no matter it is in the translation of subtitles or dubbing, the general trend is to make the language direct, popular, and easy to be understood by the general public, either with high or low educational background. Therefore, one way to achieve understandability is to simplify the language. This also 
helps with the achievement of naturalness.

Other ways to achieve it include the use of explicitation, domestication and addition, making the implicit meanings explicit, and the not understandable information understandable.

\subsubsection{L for Length}

For the unique characteristics and constraints found in ST, the final criterion proposed by the author is length, which is also one of the most important defining elements in ST. Length refers to the length of each line's translation.

The length of subtitles is constrained by the size of the TV screen and the reading speed of the audience. Too long, it would take up too much space of the screen and block the viewing of the film image. In addition, reading it would take up too much time of the audience, leaving no time for film image viewing. This is why subtitles are usually a summarization of the original line, leaving empty words such as words of mood not translated.

The length of dubbing translation is also not at the translators' free will. It must fit perfectly to the starting and finishing time of the speaker. Even though dubbing actors can adjust their speed of utterance to fit the original, most of the adjustments take place in the diction of translation. Too long, the actors' lips will have stopped moving when the dubbing voice continues. Too short, the actors' lips will still be moving when the dubbing voice has stopped, creating an uncomfortable feeling for the audience.

No matter which form is used in ST, the length of translation should always be taken into serious consideration, for the benefit of the audience.

\subsection{Summary}

The above are the eight criteria proposed by the author. The first letter of each criterion forms the English word "FANCIFUL" whose meaning also fits the characteristic of film art. To list the eight criteria together, they are:

- Faithfulness

- Acceptability

- Naturalness

- $\mathrm{C}(\mathrm{S}) y n$ chronization

- Informality Degree

- Fun

- Understandability

- Length

On the basis of the similarities found between subtitling and dubbing translation, the eight criteria include all the defining characteristics of ST. As we can see in the detailed illustrations of the eight criteria, they are in fact interrelated, yet each of these criteria has its own niche, making any of them indispensable.

The effectiveness and feasibility of this set of criteria, however, await to be tested. More researches, surveys and experiments need to be done in the testing of them. For the length limit of this paper, the author has to stop here, but further researches in this respect are expected.

\section{Conclusions}

Screen translation, as a newly evolved field of study, has not yet received enough attention from scholars. Being in its infancy, few comprehensive and systematic studies have been made. Yet with an increasing number of imported films and TV programs coming into China, more studies are desperately needed in this field. To the author's delight, this situation is undergoing a gradual change. The uniqueness of film language, now more than ever, appeals to scholars from both film study field and the translation one.

This paper is qualitative in nature and is only a tentative study in ST. Different from most previous studies, the author uses three audience-related theories as its theoretical framework, namely, reception theory, psycholinguistics and cognitive psychology. They are chosen because audience is the ultimate receiver of any audiovisual product, and their affirmation is the decisive factor in the success of any of them.

One contribution of this paper is that the author proposes seven features of film language based on Qian Shaochang's five film language features, Gottlieb's polysemiotic nature of subtitles, and the author's own observations. They are: polysemiotic, audio and visual, colloquial, variable, dramatic, transient, and noteless.

The other contribution of the author is the proposal of eight criteria customized for screen translation. These criteria are set on the basis of the similarities found between subtitling and dubbing translation. Being the two major forms of ST, their similarities represent the core features of screen translation. To help better remember these criteria, the author coins their first letters into one English word - FANCIFUL, which also reflects the characteristic of film industry. They are: Faithfulness, Acceptability, Naturalness, C(s)ynchronization, Informality degree, Fun, Understandability and Length.

This paper, however, is limited in that even though the author has tried her best to achieve objectivity, certain subjective factors can still be found in some judgments and conclusions. Many issues in ST still remain to be discussed, including the testing of the feasibility of these criteria, the translation of whispers and accents, and the translation of multilingual films.

\section{Acknowledgements}

The author would like to acknowledge that this paper is based on her dissertation for master's degree from Xi'an International Studies University, under the guidance of Prof. Han Qi. 


\section{REFERENCES}

[1] Jauß, Hans R. towards an Aesthetic of Reception, Engl. tr.by T.Bahti, 1982.

[2] Iser, Wolfgang. The Act of Reading: a Theory of Aesthetic Response. Baltimore: Johns Hopkins University Press. 1987

[3] Crosman, Robert. "Do Readers Make Meaning?" The Reader in the Text, ed. S. R. Suleiman \& I. Crosman Princeton: Princeton University Press, 1980: 149-164.

[4] Jauß, Hans R. Literaturgeschichte als Provokation. Frankfurt a.M.: Suhrkamp, 1970.

[5] Jin Yuanpu. Theory on Reception Reaction. Ninan: Shandong Education Press. 1998.

[6] Liu, Limin. Psycholinguistics. Sichuan: Sichuan University Press, 2000.

[7] Yang Peng. "A Study on Translation Process under Cognitive Psychology.” Mudanjiang Normal College Press, 2007.

[8] Wang Su, Wang Ansheng. Cognitive Psychology. Beijing: Beijing University Press, 1992.

[9] Bogucki, Łukasz. "The Constraint of Relevance in Subtitling." Journal of Specialised Translation, 1(2004): 69-85.

[10] Pavlović, Natasa. "Addressing Power and Solidarity in TV Subtitling" Across Languages and Cultures, 2004: 211232.

[11] Gottlieb, Henrik. Screen Translation. Six Studies in Subtitling, Dubbing and Voice-over. Copenhagen: Kopi Service, 2001.

[12] Sun Cuiping. "Screen Translation under Functional Equivalence." Journal of Haiyin Institute of Technology, 2018: 40.

[13] Qian, Shaochang. "The Present Status of Screen Translation in China." Meta, 1(2004): 52-8.

[14] Baker, Mona \& Malmkjær, Kirsten. Routledge Encyclopedia of Translation Studies. London: New York Taylor \& Francis Routledge, 2001. 74-6, 197-200, 244-8, 277.

[15] Gottlieb, Henrik. "Subtitling: People translating people." Subtitles, Translation \& Idioms: 1992. 83-106

[16] Fawcett, P. "Translating Film." On Translating French Literature and Film, ed. G.T.Harris. Amsterdam/Atlanta: Rodopi, 1996. 65-86.

[17] Coelh, Leonardo Jordão. "Subtitling and Dubbing: Restrictions and Priorities"Jan 24, $2008<$ http://www.tran slationdirectory.com/article326.htm>.

[18] Kilborn, Richard. " 'Speak My Language': Current Attitudes to Television Subtitling and Dubbing." Media, Culture and Society, 15(1993): 641-60 